The People's Zion 



\section{THE PEOPLE'S ZION}

SOUTHERN AFRICA, THE UNITED STATES, AND A TRANSATLANTIC FAITH-HEALING MOVEMENT

Joel Cabrita

THE BELKNAP PRESS OF HARVARD UNIVERSITY PRESS

Cambridge, Massachusetts

London, England 2018 
Copyright $\left({ }_{0} 2018\right.$ by the President and Fellows of Harvard College

All rights reserved

Printed in the United States of America

First printing

Library of Congress Cataloging-in-Publication Data

Names: Cabrita, Joel, I980- author.

Title: The people's Zion : southern Africa, the United States, and a transatlantic faith-healing movement/Joel Cabrita.

Description: Cambridge, Massachusetts : The Belknap Press of Harvard University Press, 20I 8. I Includes bibliographical references and index.

Identifiers: LCCN 2017042704 | ISBN 9780674737785 (alk. paper)

Subjects: LCSH: Zionist churches (Africa)—Africa, Southern. I Zionist churches (Africa)—

South Africa-Johannesburg. I Zionist churches (Africa) - Illinois-Zion-History-

2oth century. I Spiritual healing-Africa, Southern. I Spiritual healing-Illinois-Zion. I

Africa, Southern-Race relations. I Africa, Southern-Church history-2oth century. I

Zion (Ill.)-Church history-2oth century.

Classification: LCC BRI446 .C33 20I 8 I DDC 276.8/082-dc23

LC record available at https://lccn.loc.gov/20I7042704

Cover photograph: 'uSipho Mathunjwa noScara' (2015) (C) 2016 Sabelo Mlangeni. All rights reserved.

Cover design: Annamarie McMahon Why 
To Tarik 
